\title{
Embracing html
}

\section{Nature Chemical Biology has a new look, with more online features to facilitate the communication of chemical biology discoveries.}

As the field of chemical biology has grown, Nature Chemical Biology has expanded to reflect the diversity of science at the interface of chemistry and biology. Throughout this period, the editorial team has endeavored to publish leading research and thoughtful commentary across chemical biology and to present the field's discoveries to the widest possible audience. As we enter our sixth volume, we announce several new and upcoming features of Nature Chemical Biology papers designed to enhance these aims.

The Internet has revolutionized the ways in which we obtain information. As a society, we have welcomed online media, which provide content on demand at our computers and increasingly on our mobile devices. Many scientists are happy to read the morning news at their computers and, as a rule, access the scientific literature primarily through online versions of journals. Yet data show that most scientists download the pdf versions of research articles rather than reading the articles online in html format. Though pdfs have advantages, including a familiar layout, they generally lack the connectivity available in html articles. Given the wealth of online scientific resources and the advancement of electronic book and mobile device technology, readers will soon be able to interact fully with the online scientific literature from nearly anywhere. To support this shift in direction, Nature Chemical Biology and the other Nature journals are continuing to develop new ways to present scientific data in electronic format and to integrate resources that enhance the usability and connectivity of our published papers.

Since its inception, Nature Chemical Biology has been particularly committed to enhancing the availability of chemical information in the scientific literature. Traditionally, most published chemical content has existed as static text or imagery in journal articles. Because chemical structures and data are so central to chemical biology research, we felt that they needed to be better integrated into our papers. Simultaneously with the launch of the journal, new chemical biology initiatives, including publicly available chemical databases such as PubChem (http:// pubchem.ncbi.nlm.nih.gov), were coming online. Our desire to broaden access to and increase the usability of chemical information prompted us to develop new online chemistry functionality for the launch of the journal.

Thus, since the journal's beginnings in 2005, Nature Chemical Biology research papers have included several pioneering chemical information tools (Nat. Chem. Biol. 1, 63, 2005). First, all key chemical structures in each paper are deposited to the PubChem database as part of the publication process, which has facilitated broader communication of new compounds of interest to the scientific community. Second, readers can quickly access information on key compounds by linking to their compound pages in the html version of the paper. In 2007, we expanded these pages to include a visualization tool, featuring an energyminimized three-dimensional structure for each numbered compound (Nat. Chem. Biol. 3 , 297, 2007). Last year, chemical information in our papers became more prominent, with hover boxes for viewing chemical structures in the main text, enhanced three-dimensional viewing, and a 'compound roundup' page that collects all of the paper's key compounds in one place (Nat. Chem. Biol. 5, 1, 2009).

Over the past few months, Nature Chemical Biology has rolled out two new chemical information resources, both developed in partnership with Nature Chemistry. First, the chemical compound pages have been expanded significantly (for example, see http://www.nature.com/ nchembio/journal/v5/n12/compound/ nchembio.252_comp2b.html), providing readers with a rich resource for each compound including names, molecular properties, and chemical identifiers (such as InChI and InChIKey identifiers). Second, the html versions of Nature Chemical Biology papers now contain a highlighting tool that allows users to quickly identify additional chemical compounds in the paper and rapidly link to more information on these materials (for example, see http://www.nature.com/ nchembio/journal/v5/n12/full/nchembio.242. html). The compound pages and the annotations and their corresponding links are curated by our team of technical editors. Taken together, these two enhancements more completely integrate chemical information within each Nature Chemical Biology paper.

Several other changes are underway to enhance the experience of reading Nature Chemical Biology. Astute readers will already have noticed the first of these: beginning with this issue, the print versions and the electronic pdfs of our papers have a new look. Based on the designs of our sister Nature journals in the physical sciences, we have moved to a new journal template. We feel that this design is more engaging and that its more streamlined style improves readability and uses space more efficiently. The new template also integrates 'subject' terms, which will enhance searching and archiving of published content. Second, in the coming months, readers of Nature Chemical Biology (and the other Nature journals) will see major improvements in the html versions of online articles: article layouts and navigation are being streamlined to give our articles a new look and feel, and a variety of new tools will be introduced to enhance the reader interface and improve retrieval of other information relevant to the paper. Major changes supporting the journal web site are also in store, including enhancements to search functionality and subject archives.

Though our primary aim remains to publish the best research and insights in chemical biology, we also look forward to using new electronic publishing and social networking tools to enhance interactions in the chemical biology community. To start, Nature Chemical Biology now tweets at Twitter.com! To receive journal news regarding our latest research papers, policy updates and other information, follow @nchembio. As we continue to expand our online presence, we look to our authors and readers for feedback on how best to make use of these new technologies. We welcome your ideas electronically, but also the old-fashioned way-through interactions in person at conferences and meetings throughout 2010 and beyond. 